\title{
PRESCRIBING ZEROS OF FUNCTIONS IN THE NEVANLINNA CLASS ON WEAKLY PSEUDO-CONVEX DOMAINS IN $\mathrm{C}^{2}$
}

\author{
MEI-CHI SHAW
}

\begin{abstract}
Let $D$ be a bounded weakly pseudo-convex domain in $\mathrm{C}^{2}$ of uniform strict type. For any positive divisor $M$ of $D$ with finite area, there exists a holomorphic function $f$ in the Nevanlinna class such that $M$ is the zero set of $f$. The proof is to study the solutions of $\bar{\partial}$ with $L^{1}(\partial D)$ boundary values.
\end{abstract}

Let $D$ be a bounded weakly pseudo-convex domain in $C^{2}$. In this paper we study the Poincaré-Lelong equation

$$
i \partial \bar{\partial} u=\alpha \text { on } D
$$

where $\alpha$ is a $d$-closed $(1,1)$ form in $D$. In particular, we are interested in solutions $u$ such that the boundary values of $u$ are in $L^{1}(\partial D)$. This question arises when one tries to prescribe zeros of functions in the Nevanlinna class in $D$. It is also well known that the solutions of (1) are closely related to the solutions of the Cauchy-Riemann equation.

$$
\bar{\partial} u=f \quad \text { on } D
$$

where $f$ is a $\bar{\partial}$-closed $(0,1)$ from in $D$. When $D$ is "strongly" pseudoconvex in $\mathbf{C}^{n}, n \geq 2$, Henkin [6] and Skoda [18] have studied equations (1) and (2) independently and obtained precise estimates of the solutions $u$ on the boundary. From this they were able to completely characterize the zero sets of the functions in the Nevanlinna class on strongly pseudo-convex domains. They showed that a zero divisor is the zero set of a function in the Nevanlinna class if and only if the zero divisor satisfies the Blaschke condition (see Definitions 1.3, 1.4 in $§ \mathrm{I})$. This generalized the classical Blaschke theorem in $\mathrm{C}^{1}$ to strongly pseudo-convex domains in $\mathbf{C}^{n}$. (An excellent account of the Henkin-Skoda theorem on the unit ball in $\mathbf{C}^{n}$ can be found in Chapter 17 of Rudin's book [16], see also Harvey-Polking [5].) When $D$ is only weakly pseudo-convex, such results are unknown except for the special case when $D$ is a complex ellipsoid (see Bonami-Charpentier [2]). In this case one can use dilation to study the solutions of equations (1) and (2) from the known solutions for the ball. In this paper we solve (1) and (2) with boundary values in $L^{s}(\partial D)$ when $D$ is a

Received by the editors January 30, 1988.

1980 Mathematics Subject Classification (1985 Revision). Primary 32A10, 32A20.

Research was partially supported by NSF grant DMS 87-96300. 
weakly pseudo-convex doamin in $\mathbf{C}^{2}$ which have smoothly varying holomorphic support functions near the boundary $\partial D$. In particular our results can be applied to the case when $D$ is a convex domain with real-analytic boundary in $\mathbf{C}^{2}$. Using these results we can construct functions in the Nevanlinna class with given zeros provided the zero sets have finite area.

Our main results are the following theorems (all the definitions can be found in $\S I)$.

Theorem 1. Let $D$ be a pseudo-convex domain in $\mathbf{C}^{2}$ of uniform strict type $m$. Let $f$ be a continuous $(0,1)$-form on $\bar{D}$ and $\bar{\partial} f=0$, then there exists $a$ function $U \in \Lambda_{1 / m}(\bar{D})$ such that $\bar{\partial} u=f$ and $u$ satisfies the following estimates:

(i) $\|u\|_{L^{\prime}(D)} \leq c\left(\|f\|_{L^{1}(D)}+\|f\|_{L^{\prime}(\partial D)}\right)$,

(ii) if $p=1$,

$$
\|u\|_{L^{(m+2) /(m+1)-\varepsilon(\partial D)}} \leq c\|f\|_{L^{1}(\partial D)} \text { for every small } \varepsilon>0 \text {, }
$$

if $1<p<m+2$

$$
\|u\|_{L^{q}(\partial D)} \leq c_{p}\|f\|_{L^{p}(\partial D)} \quad \text { where } \frac{1}{q}=\frac{1}{p}-\frac{1}{m+2},
$$

if $p=m+2$

$$
\|u\|_{L^{p^{\prime}(\partial D)}} \leq c_{p}\|f\|_{L^{p}(\partial D)} \text { for all } p^{\prime}<\infty
$$

if $m+2<p \leq \infty$

$$
\|u\|_{\Lambda_{1 / m-(m+2) / m p}(\partial D)} \leq c_{p}\|f\|_{L^{p}(\partial D)},
$$

(iii) $\|u\|_{\Lambda_{1 / m}^{p}(\partial D)} \leq c_{p}\|f\|_{L^{p}(\partial D)}$ for every $1 \leq p \leq \infty$ where $c$, $c_{p}$ are constants depending only on $D, m, p$.

Let $H^{k}(D, R)$ be the DeRham cohomology of degree $k$ on $D$. We have the following theorem on the solutions of equation (1).

Theorem 2. Let $D$ be a pseudo-convex domain in $\mathbf{C}^{2}$ of uniform strict type $m$ and $H^{2}(D, R)=0$. Let $\alpha$ be a positive $d$-closed $(1,1)$ form on $D$ with smooth

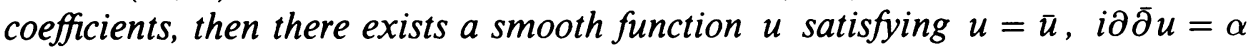
and

$$
\|u\|_{L^{(m+2) /(m+1)-\varepsilon(\partial D)}}+\|u\|_{L^{\prime}(D)} \leq C\|\alpha\|_{L^{\prime}(D)}
$$

for any $\varepsilon>0$ small.

Let $H^{2}(D, Z)$ be the second Čech cohomology group with integer coefficients, then using Theorems 1 and 2 we have

Theorem 3. Let $D$ be a pseudo-convex domain in $\mathbf{C}^{2}$ of uniform strict type and $H^{2}(D, Z)=0$. Let $M$ be a positive divisor of $D$ such that $M$ has finite area, then there exists a holomorphic function $f$ in the Nevanlinna class such that $M$ is the zero set of $f$.

When $D$ is strongly pseudo-convex, this result was obtained by Gruman [4]. 
In [20]. Stein showed that for any function $f$ in the Nevanlinna class on $D, f$ has admissible limits at almost every boundary point. Using this and Theorem 3, we have the following result concerning the boundary values of meromorphic functions of bounded characteristic.

Corollary 3.1. Let $D$ be the same as in Theorem 3. Let $g$ be a meromorphic function of bounded characteristic in $D$ such that the pole sets of $g$ have finite area. Then one can find two holomorphic functions $f$ and $h$ in the Nevanlinna class on $D$ such that $g=f / h$. In particular, $g$ has admissible limits at almost every boundary points.

The plan of the paper is as follows: In $\S 1$ we introduce the notation which is used in the paper. Theorem 1 is proved in $\S I I$ using the Henkin kernel. The Henkin kernel for the domains we considered was constructed by Range [14]. Our main task is to relate the boundary values of the solution to the solution of the tangential Cauchy-Riemann equation $\bar{\partial}_{b}$ obtained by the author recently in [17], thus providing the estimates on the boundary. We prove Theorems 2 and 3 in §III.

\section{NOTATION AND PRELIMINARIES}

Let $D$ be a bounded domain in $\mathbf{C}^{2}$ with smooth boundary $\partial D$. Let $\rho$ be a defining function for $D$ such that $D=\left\{z \in \mathbf{C}^{2} \mid \rho(z)<0\right\}$ and $|d \rho| \neq 0$ on $\partial D$. In particular we shall take $\rho=-\operatorname{dist}(z, \partial D)$ when $z \in D$ and $\rho=\operatorname{dist}(z, \partial D)$ when $z \notin D$. If $\delta>0$ is small, we define $D^{\delta}=\left\{z \in \mathbf{C}^{2} \mid\right.$ $\rho(z)<\delta\}$ and $U^{\delta}=D^{\delta} \backslash D . D_{\delta}=\left\{z \in \mathbf{C}^{2} \mid \rho(z)<-\delta\right\}$. The Lebesgue measure on $D$ is denoted by $d V$ the surface measure on $\partial D$ and $\partial D_{\delta}$ is denoted by $d S, d S_{\delta}$ respectively. The letter $c$ will always denote a positive constant which can vary from line to line.

Definition 1.1. $D$ is pseudo-convex if on $\partial D$ we have $\langle\partial \bar{\partial} \rho, L \wedge \bar{L}\rangle \geq 0$ where $L$ is a nonzero tangential holomorphic vector field.

Definition 1.2. A domain $D$ is called pseudo-convex of uniform strict type $m$, $m \in N$, if $D$ is pseudo-convex and there exists a $C^{1}$ function $F(\zeta, z)$ on $U^{\delta} \times D^{\delta}$ such that

(i) $F(\zeta, \cdot)$ is holomorphic on $D$,

(ii) $F(\zeta, \zeta)=0,\left.d_{z} F\right|_{\zeta=z}=\partial \rho(\zeta) \neq 0$,

(iii) $\rho(z)-\rho(\zeta) \geq c|\zeta-z|^{m}$ for $\zeta \in U^{\delta},|z-\zeta|<\varepsilon$ and $F(\zeta, z)=0$ where $c$ and $\varepsilon$ are some positive constants.

Thus, if a domain $D$ is of uniform strict type $m$, then there exists a smoothly varying holomorphic support function near the boundary, and the holomorphic curve defined by the zeros of the support function has order of contact $m$ with the boundary. (For related properties of uniform strict type, see Kohn [8] and Range [14].) Simple examples of pseudo-convex domains of uniform strict type are strongly pseudo-convex domains $(m=2)$, domains of strict type defined in 
Kohn [8] and the convex domains with real-analytic boundaries. If a pseudoconvex domain is of uniform strict type $m$, then $m$ must be even. We note that there are pseudo-convex domains of finite type in $\mathbf{C}^{2}$ without holomorphic support functions (see Kohn-Nirenberg [9]).

Let $C^{k}(D)$ be the space of functions whose $k$ th derivatives are continuous on $D, 0 \leq k \leq \infty . L^{s}(D), 1 \leq s<\infty$, denotes the space of functions whose $s$ th power are absolutely integrable. As usual $L^{\infty}(D)$ denotes the space of bounded functions. Let $L(D)$ be the space of finite regular measures on $D$. If $u \in L(D)$ then we define $|u|_{L(D)}$ to be the total mass $u(D)$. Any $(p, q)$ form $\alpha$ on $D$ is said to be in $C^{k}(\bar{D}), L^{s}(D)$ or $L(D)$, denoted by $C_{p, q}^{k}(D), L_{p, q}^{s}(D)$ and $L_{p, q}(D)$, if its coefficients are in those spaces and the norm of a form is the sum of the norm of each coefficient. The functions spaces $C^{k}(\partial D), L^{2}(\partial D)$ and $L(\partial D)$ are defined similarly. We define the spaces $A_{\alpha}^{p}(\partial D), 0<\alpha<1$, $1 \leq p \leq \infty$, to be the function space with the following norm (see Stein [19])

$$
\|u\|_{\Lambda_{t}^{p}(\partial D)}=\|u\|_{L^{p}(\partial D)}+\sup _{\substack{x(t) \in \mathbf{C} \\ 0 \leq t \leq}} \frac{\| u\left(x(t)-u(x(0)) \|_{L^{p}(\partial D)}\right.}{|t|^{\alpha}}<\infty
$$

where $\mathbf{C}=\left\{X(t)=t \in[0,1] \rightarrow X(t) \in \partial D, X(t)\right.$ is $C^{1}$ and $\left.\left|X^{\prime}(t)\right| \leq 1\right\}$. When $p=\infty$, we write $\Lambda_{\alpha}^{\infty}(\partial D)=\Lambda_{\alpha}(\partial D)$ also, which is the Hölder space of exponent $\alpha$. It is well known that $\Lambda_{\alpha}^{p}(\partial D) \subset L^{p}(\partial D)$ and the inclusion is compact.

The $(p, q)$ forms on $\partial D$, denoted by $B_{p, q}(\partial D)$, are the projection of the $(p, q)$ forms on $D$ onto the parts which are orthogonal to $\bar{\partial} \rho$. We use $\tau$ to denote this projection. The tangential Cauchy-Riemann operators $\bar{\partial}_{b}=B_{p, q}(\partial D) \rightarrow B_{p, q+1}(\partial D)$ are the induced complex of the Cauchy-Riemann complex $\bar{\partial}$ on $D$. We refer the readers to Kohn-Rossi [10] for the basic properties of $\bar{\partial}_{b}$.

A $(p, q)$ current on $D$ is a $(p, q)$ form with distribution coefficients. A (1.1) current $\alpha$ is called positive if for every compactly supported $C_{0,1}^{\infty}(D)$ from $\omega$, we have

$$
\int_{D} \alpha \wedge\left(\frac{\omega \wedge \bar{\omega}}{i}\right) \geq 0
$$

It is proved in Lelong [12] that if a (1.1) current $\alpha=\sum_{i, j=1}^{2} \alpha_{i j} d z_{i} \wedge d \bar{z}_{j}$ is positive, then $\alpha=\bar{\alpha}, \alpha_{i j}=-\alpha_{j i}$ and all the $\alpha_{i j}$ are locally finite measures. It also follows from Lelong [11] that the zero divisor $M_{f}$ of a holomorphic function $f$ on $D$ induces a (1.1) $d$-closed positive current, denote by $\left[M_{f}\right]$ on $D$. For details of these matters, see Lelong [11].

Let $M$ be a positive divisor in $D$ such that $M=\gamma_{j} M_{j}$ where $M_{j}$ 's are irreducible 1-dimensional complex variety and $\gamma_{j}$ is a positive integer. If we assume $H^{2}(D, Z)=0$, then there exists a holomorphic function $f$ such that $f$ determines $M$, i.e., $f$ vanishes on $M_{j}$ to order $\gamma_{j}$. 
Definition 1.3. A divisor $M=\sum \gamma_{j} M_{j}$ is said to have finite area if

$$
\sum_{j} \gamma_{j} \int_{z \in M_{j}} d \delta(z)<\infty
$$

$M$ is said to satisfy the Blaschke condition if

$$
\sum_{j} \gamma_{j} \int_{z \in M_{j}}|\rho(z)| d \delta(z)<\infty
$$

where $d \delta(z)$ is the area measure on $M_{j}$.

Definition 1.4. A holomorphic function $f$ on $D$ is in the Nevanlinna class, denoted by $N(D)$, if $f$ satisfies

$$
\sup _{\varepsilon \rightarrow 0} \int_{\partial D_{\varepsilon}} \log ^{+}|f(z)| d f S_{\varepsilon}(z)<\infty
$$

where $\log ^{+}|f(z)|=\max \{\log |f(z)|, 0\}$ and $d S_{\varepsilon}$ is the surface element of $\partial D_{\varepsilon}$.

Definition 1.5. A meromorphic function $g$ is in the Nevanlinna class $N(D)$ (or of bounded characteristic) if

$$
\sup _{\varepsilon \rightarrow 0^{+}} \int_{\partial D_{\varepsilon}} \log ^{+}|g(z)| d S_{\varepsilon}(z)<\infty
$$

and the pole set of $g$ satisfying the Blaschke condition. (see Nevanlinna [13], Stoll [21])

It follows from Henkin [6], Skoda [18] that if $f \in N(D)$, then the zero divisor $M_{f}$ of $f$ must satisfy the Blaschlse condition. As Skoda pointed out in [18] that this condition was first observed by Stoll and Gavot.

\section{SOLVING $\bar{\partial}$ WITH $L^{p}$ ESTIMATES ON THE BoundaRY}

In this section we shall prove the estimates in Theorem 1 using Henkin's solution for $\bar{\partial}$ constructed by Range [14]. For the basic properties of Henkin kernel, we refer the readers to Henkin-Leiterer [7] and Range [14] and Romanov [15].

Proof of Theorem 1. By the assumption of uniform strict type $m$, it is proved in $[14,17]$ that there is exists a $C^{1}$ function $\Phi(\zeta, z)$ on $U^{\delta} \times D^{\delta}$ which is holomorphic in $z \in D^{\delta}$ and $\Phi$ satisfies

(i) $\Phi(\zeta, \zeta)=0, \Phi(\zeta, \zeta) \neq 0$ for all $|\zeta-z| \geq \varepsilon$,

(ii) $\Phi(\zeta, z)=F(\zeta, z) H(\zeta, z)$ for $|\zeta-z|<\varepsilon$, where $H$ is a $C^{1}$ function with $0<A_{0} \leq|H| \leq A_{1}<\infty$.

By Hefer's theorem, we also have the decomposition

$$
\Phi(\zeta, z)=\sum_{i=1}^{2} P_{i}(\zeta, z)\left(\zeta_{i}-z_{i}\right)=\langle P(\zeta), \zeta-z\rangle
$$


where the functions $P_{i}(\zeta, z)$ are $C^{1}$ in $\zeta$ and holomorphic in $z, P(\zeta, z)=$ $\left(P_{1}(\zeta, z), P_{2}(\zeta, z)\right)$ is the Leray map which is holomorphic in $z$.

It is well known that from $P(\zeta, z)$ and the Bochner-Martinelli-Koppelman formula one can construct the Henkin kernel for the $\bar{\partial}$-equation. Let

$$
B(\zeta, z)=\frac{1}{(2 \pi i)^{2}} \frac{\left(\bar{\zeta}_{1}-z_{1}\right) d \bar{\zeta}_{2}-\left(\bar{\zeta}_{2}-z_{2}\right) d \bar{\zeta}_{1}}{|\zeta-z|^{4}}
$$

be the Bochner-Martinelli kernel and

$$
L(\zeta, z)=\frac{1}{(2 \pi i)^{2}} \frac{P_{1}(\zeta, z) \bar{\partial}_{\zeta, z} P_{2}(\zeta, z)-P_{2}(\zeta, z) \bar{\partial}_{\zeta, z} P_{1}(\zeta, z)}{\langle P(\zeta, z), \zeta-z\rangle^{2}}
$$

and

$$
\begin{aligned}
R(\zeta, z, \lambda)=\frac{1}{(2 \pi i)^{2}}\left[\eta _ { 1 } ( \zeta , z , \lambda ) \wedge \left(\bar{\partial}_{\zeta, z}\right.\right. & \left.+d_{\lambda}\right) \eta_{2}(\zeta, z, \lambda) \\
& \left.-\eta_{2}(\zeta, z, \lambda) \wedge\left(\bar{\partial}_{\zeta, z}+d_{\lambda}\right) \eta_{1}(\zeta, z, \eta)\right]
\end{aligned}
$$

where

$$
\eta_{j}(\zeta, z, \lambda)=\lambda \frac{\bar{\zeta}_{j}-\bar{z}_{j}}{|\zeta-z|^{2}}+(1-\lambda) \frac{P_{j}(\zeta, z)}{\langle P(\zeta, z), \zeta-z\rangle}, \quad j=1,2
$$

We define for $f \in C_{0,1}(\bar{D}), z \in D$

$$
B_{D} f(z)=\int_{D} f(\zeta) \wedge B(\zeta, z) \wedge d \zeta_{1} \wedge d \zeta_{2}
$$

and

$$
R_{\partial D} f(z)=\int_{\substack{\partial D \\ 0 \leq \lambda \leq 1}} f(\zeta) \wedge R(\zeta, z, \lambda) \wedge d \zeta_{1} \wedge d \zeta_{2}
$$

Integrating over $\lambda$, we have

$$
R_{\partial D} f(z)=\int_{\partial D} f(\zeta) \wedge K(\zeta, z) \wedge d \zeta_{1} \wedge d \zeta_{2}
$$

where

$$
K(\zeta, z)=\frac{1}{(2 \pi i)^{2}} \frac{P_{1}(\zeta, z)\left(\bar{\zeta}_{2}-\bar{z}_{2}\right)-P_{2}(\zeta, z)\left(\bar{\zeta}_{1}-\bar{z}_{1}\right)}{\Phi(\zeta, z)|\zeta-z|^{2}}
$$

It follows from the construction of Henkin that $u=B_{D} f(z)+R_{\partial D} f(z)$ is a solution to the equation $\bar{\partial} u=f$ on $D$ and $u \in \Lambda 1_{1 / m}(D)$ (see p. 249 in Range [14]). To prove the estimate (i) in Theorem 1, we estimate $B_{D} f$ and $R_{\partial D} f$ separately.

Since $|B(\zeta, z)| \leq c /|\zeta-z|^{3}$, it is easy to see $B(\zeta, z)$ is a kernel of weak type 4/3. (For definition of weak type, see Folland-Stein [3].) Thus $B_{D} f$ is a bounded operator from $L^{1}(D)$ to $L^{4 / 3-\varepsilon}(D)$ for every small $\varepsilon>0$. In particular, $\left\|B_{D} f\right\|_{L^{\prime}(D)} \leq C\|f\|_{L^{\prime}(D)}$. 
To estimate $R_{\partial D} f$, we notice that for fixed $\zeta \in \partial D$, the kernel $K(\zeta, z)$ has singularity only when $z=\zeta$. If we fix a ball $B_{\varepsilon}(\zeta)$, centered at $\zeta$ with radius $\varepsilon$, it is easy to see

$$
\int_{D \backslash B_{\varepsilon}(\zeta)}|K(\zeta, z)| d V(z) \leq C \int_{D \backslash B_{\varepsilon}(\zeta)} \frac{1}{|\Phi(\zeta, z)||\zeta-z|} d V(z)<C
$$

uniformly in $\zeta$. Thus we only need to estimate $\int_{B_{\varepsilon}(\zeta)}|K(\zeta, z)| d V(z)$. On $B_{\varepsilon}$ we introduce a special coordinate chart $\left(t_{1}, t_{2}, t_{3}, y\right)=(t, y)$ such that $y=|\rho(z)|, t_{3}=\operatorname{Im} \Phi(\zeta, z)$ and $\left(t_{1}, t_{2}\right)=t^{\prime}$ is chosen so that $t^{\prime}(z)=t^{\prime}(\hat{z})$ where $\hat{z} \in \partial D$ is the normal projection of $z$ onto $\partial D$. It follows from [17, Lemma 3.3] that $|\Phi(\zeta, z)| \geq c\left(|\zeta-z|^{m}+|\rho(z)|+|\operatorname{Im} \Phi(\zeta, z)|\right)$. Thus under the special coordinate chart, we have $|\Phi(\zeta, z)| \geq c\left(\left|t^{\prime}\right|^{m}+\left|t_{3}\right|+y\right)$. We choose a large number $R$, then

$$
\begin{aligned}
\int_{D \cap B_{c}(\zeta)}|K(\zeta, z)| d V(z) & \leq \int_{|(t, y)| \leq R} \frac{1}{\left(\left|t^{\prime}\right|^{m}+\left|t_{3}\right|+y\right)\left(\left|t^{\prime}\right|\right)} d t_{1} d t_{2} d t_{3} d y \\
& \leq C
\end{aligned}
$$

independent of $\zeta$.

Using (2.2), (2.3), we have

$$
\begin{aligned}
\left\|R_{\partial D} f\right\|_{L^{\prime}(D)} & \leq \int_{D} \int_{\partial D}|K(\zeta, z) \| f(\zeta)| d S(\zeta) d V(z) \\
& \leq \int_{\partial D}\left(\int_{D}|K(\zeta, z)| d V(z)|f(\zeta)|\right) d S(\zeta) \\
& \leq c \int_{\partial D}|f(\zeta)| d S(\zeta)=c\|f\|_{L^{1}(\partial D)} .
\end{aligned}
$$

Combining (2.1) and the above, we have proved (i).

In order to estimate the boundary values of $u$ in terms of the boundary values of $f$, we express the integral $B_{D} f$ in a different manner using $L(\zeta, z)$ when $z \in \partial D$.

Introducing kernels $R^{*}(\zeta, z, \lambda), K^{*}(\zeta, z)$ and $L^{*}(\zeta, z)$ where

$$
R^{*}(\zeta, z, \lambda)=R(z, \zeta, \lambda), \quad K^{*}(\zeta, z)=K(z, \zeta), \quad L^{*}(\zeta, z)=L(z, \zeta),
$$

then $R^{*}(\zeta, z, \lambda), K^{*}(\zeta, z)$ and $L^{*}(\zeta, z)$ are well defined for $\zeta \in D$ and $z \in$ $U^{\delta}$. Since $R(\zeta, z, \lambda)$ satisfies the homotopy formula (see Lemma 2.5 in Shaw [17] for a proof) $\left(\partial_{\zeta, z}+d_{\lambda}\right) R(\zeta, z, \lambda)=0$ for $z \in D, \zeta \in U^{\delta}$, we have

$$
\left(\bar{\partial}_{\zeta, z}+d_{\lambda}\right) R^{*}(\zeta, z, \lambda)=0 \text { for } \zeta \in D, z \in U^{\delta} .
$$


For $z \in U^{\delta}$, it follows from (2.4) and the fact $L^{*}(\zeta, z)$ is holomorphic in $\zeta \in D$, we have by Stokes' theorem,

$$
\begin{aligned}
\int_{\substack{\zeta \in D \\
0 \leq \lambda \leq 1}} f(\zeta) \wedge \bar{\partial}_{\zeta} R^{*}(\zeta, z, \lambda) \wedge d \zeta_{1} \wedge d \zeta_{2} \\
=\int_{\zeta \in D} f(\zeta) \wedge \bar{\partial}_{\zeta, z} R^{*}(\zeta, z, \lambda) \wedge d \zeta_{1} \wedge d \zeta_{2} \\
=\int_{\substack{\zeta \in \lambda \leq 1 \\
0 \leq \lambda \leq 1}} d_{\lambda}\left[f(\zeta) \wedge R^{*}(\zeta, z, \lambda) \wedge d \zeta_{1} \wedge d \zeta_{2}\right] \\
=\left.\int_{\zeta \in D} f(\zeta) \wedge R^{*}(\zeta, z, \lambda) \wedge d \zeta_{1} \wedge d \zeta_{2}\right|_{\lambda=0} ^{\lambda=1} \\
=\int_{\zeta \in D} f(\zeta) \wedge\left[B(\zeta, z)-L^{*}(\zeta, z)\right] \wedge d \zeta_{1} \wedge d \zeta_{2} \\
=\int_{\zeta \in D} f(\zeta) \wedge B(\zeta, z) \wedge d \zeta_{1} \wedge d \zeta_{1} .
\end{aligned}
$$

Thus for $z \in \partial D$, we have $\bar{\partial} f=0$

(2.5)

$$
\begin{aligned}
\int_{\zeta \in D} f(\zeta) \wedge B(\zeta, z) \wedge d \zeta_{1} \wedge d \zeta_{2} & =-\int_{\substack{\zeta \in D \\
0 \leq \lambda \leq 1}} \bar{\partial}_{\zeta}\left[f(\zeta) \wedge R^{*}(\zeta, z, \lambda)\right] \wedge d \zeta_{1} \wedge d \zeta_{2} \\
& =-\int_{\substack{\zeta \in \partial D \\
0 \leq \lambda \leq 1}} f(\zeta) \wedge R^{*}(\zeta, z, \lambda) \wedge d \zeta_{1} \wedge d \zeta_{2}
\end{aligned}
$$

Substituting (2.5) in $u$ we obtained the following formula for $u$ if $z \in \partial D$ :

$$
\begin{aligned}
u(z)= & \int_{\substack{\zeta \in \partial D \\
0 \leq \lambda \leq 1}} f(\zeta) \wedge R(\zeta, z, \lambda) \wedge d \zeta_{1} \wedge d \zeta_{1} \\
& -\int_{\substack{\zeta \in \partial D \\
0 \leq \lambda \leq 1}} f(\zeta) \wedge R^{*}(\zeta, z, \lambda) \wedge d \zeta_{1} \wedge d \zeta_{2} \\
\equiv & R_{\partial D} f(z)-R_{\partial D}^{-} f(z) .
\end{aligned}
$$

Let $f(z)=f_{t}+f_{n}$ where $f_{t}=\tau f$ on $\partial D$ where $\tau$ is defined in $\S I$ and $f_{n}=g \bar{\partial} \rho$ for some function $g$ on $\partial D$. Then

$$
\begin{aligned}
R_{\partial D} f_{n} & =\int_{\zeta \in \partial D} g(\zeta) \wedge \bar{\partial} \rho \wedge K(\zeta, z) \wedge d \zeta_{1} \wedge d \zeta_{2} \\
& =\int_{\zeta \in \partial D} g(\zeta) \wedge d \rho \wedge K(\zeta, z) \wedge d \zeta_{1} \wedge d \zeta_{2} \\
& =0
\end{aligned}
$$

Thus $R_{\partial D} f(z)=R_{\partial D} f_{t}(z)$. Similarly $R_{\partial D}^{-} f(z)=R_{\partial D}^{-} f_{t}(z)$. Thus we have

$$
u(z)=R_{\partial D} f_{t}(z)-R_{\dot{\partial} D}^{-} f_{t}(z)
$$


i.e. the boundary values of $u$ depends only on the tangential part of $f$ on $\partial D$. Formulas $(2.6)$ coincides with the formula (2.15) in [17], which solves the tangential Cauchy-Riemann equation $\bar{\partial}_{b} u=f_{t}$ on $\partial D$.

It is proved in Lemma 3.22 of [17] that the kernels $K(\zeta, z)$ and $K^{*}(\zeta, z)$ are of weak type $\frac{m+2}{m+1}$ on $\partial D$. Thus we have all the results in (ii). The estimates (iii) follows from Theorem 2 in [17]. Thus our theorem is proved.

\section{Poincare-Lelong eQuation AND ITS APPLiCATIONS}

In this section we shall prove the estimates for the Poincaré-Lelong equation and its applications (Theorems 2 and 3). To prove Theorem 2 we decompose Equation (1) into two steps, first applying the Poincare lemma and then solving a $\bar{\partial}$-equation using Theorem 1 . Theorem 3 follows from Theorems 1 and 2 using an approximation argument and the Poincaré-Lelong formula.

Proof of Theorem 2. We first assume $D$ is star-shaped and $0 \in D$. Using Poincaré lemma, we define

$$
\begin{aligned}
P \alpha(\zeta)= & \sum_{j}\left(\sum_{i} \int_{0}^{1} t \alpha_{i j}(t z) d t z_{i}\right) d \bar{z}_{j} \\
& -\sum_{i}\left(\sum_{j} \int_{0}^{1} t \alpha_{i j}(t z) d t \bar{z}_{j}\right) d z_{i}
\end{aligned}
$$

then $d P \alpha(z)=\alpha(z)$. Since $\alpha$ is positive, it follows from Lelong that $\alpha_{j i}=$ $-\alpha_{i j}$. Thus we define

$$
f(z)=\sum_{j}\left(\sum_{i} \int_{0}^{1} t \alpha_{i j}(t z) d t z_{i}\right) d \bar{z}_{j}
$$

then $P \alpha(z)=f(z)+\bar{f}(z)$ and we have $\bar{\partial} f=\partial \bar{f}=0$. Furthermore,

$$
\begin{aligned}
\|f\|_{L^{\prime}(\partial D)} & \leq \sum_{i j} \int_{\partial D}\left(\int_{0}^{1}\left|\alpha_{i j}(t z)\right| d t\right) d S \\
& \leq c \sum_{1, j} \int_{D}\left|\alpha_{i j}(z)\right| d V(z)=c|\alpha|_{L^{1}(D)}
\end{aligned}
$$

It is easy to see $\|f\|_{L^{\prime}(D)} \leq c\|\alpha\|_{L^{\prime}(D)}$.

Thus applying Theorem 1 , we can find a function $v$ satisfying $\bar{\partial} v=f$ and the estimates

$$
\begin{gathered}
\|v\|_{L^{\prime}(D)}+\|v\|_{L}^{(m+2) /(m+1)-\varepsilon}(\partial D) \leq c\left(\|f\|_{L^{\prime}(D)}+\|f\|_{L^{\prime}(\partial D)}\right) \\
\leq c\|\alpha\|_{L^{\prime}(D)} .
\end{gathered}
$$

Since

$$
\alpha=d(f+\bar{f})=\partial f+\bar{\partial} \bar{f}=\partial(\bar{\partial} v)+\bar{\partial}(\partial \bar{v})=i \partial \bar{\partial}\left(\frac{v-\bar{v}}{i}\right)
$$


Let $u=(v-\bar{v}) / i$, then $u=u$ and Theorem 2 is proved when $D$ is star-shaped. For the general case when $D$ is not star-shaped, by the assumption $H^{2}(D, R)=0$, one can use the well-known global construction of Weil for the DeRham cohomology and the theorem is proved.

Proof of Theorem 3. Let $\alpha_{M}$ be the closed (1.1) current associated with the zero set $M$, i.e., if $h$ is a holomorphic function on $D$ which has zero set $M$, then $\frac{i}{\pi} \partial \bar{\partial} \log |h|=\alpha_{M}$ by the Poincaré-Lelong formula. Let $\varphi_{\varepsilon}(x) \in C_{0}^{\infty}(R)$ be a family of functions such that $\varphi_{\varepsilon}(x) \geq 0$ for all $x \in R, \varphi_{\varepsilon}(x)$ is supported on $|x| \leq \varepsilon / 2$ and $\int_{-\infty}^{\infty} \varphi_{\varepsilon}(x) d x=1$. We define $V_{\varepsilon}(z)=\log |h| * \varphi_{\varepsilon}$, it follows that $V_{\varepsilon}(z) \in C^{\infty}\left(D_{\varepsilon}\right)$ and $V_{\varepsilon}(z) \rightarrow \log |h(z)|$. We define $\widetilde{V}_{\varepsilon}(z)$ to be a smooth extension of $V_{\varepsilon}(z)$ to a neighborhood of $D$ then $\widetilde{V}_{\varepsilon}(z)$ is a smooth function on $\bar{D}$ and $\widetilde{V}_{\varepsilon}(z) \rightarrow \log |h(z)|$ a.e.

We define $\alpha_{\varepsilon}=\frac{1}{\pi} \partial \tilde{\partial} \widetilde{V}_{\varepsilon}$, it follows that $\alpha_{\varepsilon} \in C_{1,1}^{\infty}(\bar{D}), \alpha_{\varepsilon}$ is $d$-closed and positive.

In particular, $\alpha_{\varepsilon}=\sum_{i, j=1}^{2} \alpha_{i j}^{\varepsilon} d z_{i} \wedge d \bar{z}_{j}, \alpha_{\varepsilon} \rightarrow \alpha$ in measure $\alpha_{i j}^{\varepsilon} \leq \alpha_{i i}^{\varepsilon}+\alpha_{j j}^{\varepsilon}$. Thus there exists a constant $A$ such that

$$
\left\|\alpha_{\varepsilon}\right\|_{L^{\prime}(D)} \leq C \sum_{i, j=1}^{2}\left\|\alpha_{i j}^{\varepsilon}\right\|_{L^{\prime}(D)} \leq C \sum_{i}\left\|\alpha_{i i}^{\varepsilon}\right\|_{L^{\prime}(D)}<A .
$$

Applying Theorem 2 to $\alpha_{\varepsilon}$, we can find a smooth $u_{\varepsilon}=\bar{u}_{\varepsilon}, \frac{1}{\pi} \partial \bar{\partial}_{\varepsilon}=\alpha_{\varepsilon}$ and

$$
\left\|u_{\varepsilon}\right\|_{L^{1}(\partial D)}+\left\|u_{\varepsilon}\right\|_{L^{1}(D)} \leq C\left\|\alpha_{\varepsilon}\right\|_{L^{\prime}(D)} \text {. }
$$

We define $g_{\varepsilon}=u_{\varepsilon}-\bar{v}_{\varepsilon}$, then $g_{\varepsilon}$ is pluriharmonic on $D$. Thus $g_{\varepsilon}$ is the real part of a holomorphic function. Since $\log |h|$ is plurisubharmonic, it is locally integrable. We have that for any compact subset $K$ of $D$, there exists a constant $C_{K}$.

$$
\int_{K}\left|v_{\varepsilon}\right| d V<C_{K} \quad \text { uniformly in } \varepsilon .
$$

Following (3.1), (3.2), we also have for some constant $C$

$$
\int_{D}\left|u_{\varepsilon}\right| d V<C \quad \text { uniformly in } \varepsilon
$$

Thus for every compact subset $K$ of $D$, there exists a constant $C$ such that

$$
\int_{K}\left|g_{\varepsilon}\right| d V<C \quad \text { uniformly in } \varepsilon \text {. }
$$

It follows that $g_{\varepsilon}$ is uniformly bounded on every subset of $D$. Thus it is a normal family and there exists a convergent subsequence of $g_{\varepsilon}$ that converges to a function $g$ uniformly on compact subsets of $D$. The function $g$ is also pluriharmonic.

Let $u=\log |h|+g$, then $u(z)=\lim u_{\varepsilon}(z)$ and $u \in L^{1}(D)$ and $u$ satisfies $\frac{1}{\pi} \partial \partial u=\alpha_{M}$ in the distribution sense. Using Theorem 1, we have

$$
\left\|u_{\varepsilon}\right\|_{\Lambda_{1} / m(\partial D)} \leq C\left\|\alpha_{\varepsilon}\right\|_{L^{\prime}(D)} \leq A,
$$


there exists a subsequence of $u_{\varepsilon}$ that converges strongly in $L^{1}(\partial D)$ to $u$ and we have $\int_{\partial D}|u(z)| d s<\infty$. Since $g=\operatorname{Re} G$ for some holomorphic function $G$ on $D$, we can write

$$
u=\log |h|+\operatorname{Re} G=\log \left|h e^{G}\right| .
$$

Let $f=h e^{G}$, it follows from Poincare-Lelong equation the zero set of $f$ is the same as $h$ and $f \in N(D)$. Thus the theorem is proved.

Proof of Corollary 3.1. Let $g=\tilde{f} / \tilde{h}$ for some holomorphic function $\tilde{f}$ and $\tilde{h}$. By assumption the zero set of $\tilde{h}$, has finite area. Thus by Theorem 3 there exists a function $h \in N(D)$ such that $\tilde{h}=h Q$ for some holomorphic function $Q$ which never vanishes on $D$. Let $f=g \cdot h$, then it is easy to see that $f$ is holomorphic in $D$ and

$$
\begin{aligned}
\sup _{\varepsilon} \int_{\partial D_{\varepsilon}} \log ^{+}|f(z)| d S_{\varepsilon} \leq & \sup _{\varepsilon} \int_{\partial D_{\varepsilon}} \log ^{+}|g(z)| d S_{\varepsilon} \\
& +\sup _{\varepsilon} \int_{\partial D_{\varepsilon}} \log ^{+}|h(z)| d S_{\varepsilon}<\infty .
\end{aligned}
$$

Using Theorem II, p. 48 in Stein [20], both $f$ and $h$ have nonzero admissible limits at almost every boundary point, it follows that $g$ has admissible limits at almost every boundary point.

Remark. When $D$ is a ball in $\mathbf{C}^{2}$, under the same assumption as in Theorem 3, Berndtsson [1] has proved that there exists a bounded holomorphic function with zeros $M$. It is still unknown if this is true even for the general strongly pseudo-convex domains in $\mathbf{C}^{2}$.

\section{REFERENCES}

1. B. Berndtsson, Integral formulas for the $\partial \bar{\partial}$-equation and zeros of bounded holomorphic functions in the unit ball, Math. Ann. 249 (1980), 163-176.

2. A. Bonami and P. Charpentier, Solutions de l'equation a et zéros de la class de Nevanlinna dans certains domaines faiblement pseudo-convexes, Ann. Inst. Fourier (Grenoble) 32 (1982), 53-89.

3. G. B. Folland and E. M. Stein, Estimates for the $\bar{\partial}_{b}$ complex and analysis on the Heisenberg group, Comm. Pure Appl. Math. 27 (1974), 429-522.

4. L. Gruman, The zeros of holomorphic functions in strictly pseudo-convex domains, Trans. Amer. Math. Soc. 207 (1975), 163-174.

5. F. R. Harvey and J. C. Polking, The J-Neumann solution to the inhomogeneous CauchyRiemann equation in the ball in $\mathbf{C}^{n}$, Trans. Amer. Math. Soc. 281 (1984), 587-613.

6. G. M. Henkin, Lewy's equation and analysis on a pseudo-convex manifold. II, Math. USSR Sb. 31 (1977), 63-94.

7. G. M. Henkin and J. Leiterer, Theory of functions on complex manifolds, Birkhäuser, Boston, Mass., 1984.

8. J. J. Kohn, Boundary behavior of $\bar{\partial}$ on weakly pseudo-convex manifolds of dimension two, J. Differential Geom. 6 (1972), 523-542.

9. J. J. Kohn and L. Nirenberg, A pseudo-convex domain not admitting a holomorphic support function, Math. Ann. 201 (1973), 265-268. 
10. J. J. Kohn and H. Rossi, On the extension of holomorphic functions from the boundary of a complex manifold, Ann. of Math. 81 (1965), 451-472.

11. P. Lelong, Intégration sur un ensemble analytique complexe, Bull. Soc. Math. France 85 (1957), 239-262.

12. P. Lelong, Fonctions plurisousharmoniques et formes différentielles positives, Gordon and Breach, New York; Dunod, Paris, 1968.

13. R. Nevanlinna, Eindeutige analytische Funktionen, Springer-Verlag, Berlin, 1963; English transl. 1970.

14. R. M. Range, On Hölder estimates for $\bar{\partial} u=f$ on weakly pseudo-convex domains, Proc. Internat. Conf. (Cortona, Italy), Scuola Norm. Sup. Pisa, 1978, pp. 247-267.

15. A. V. Romanov, A formula and estimates for solutions of the tangential Cauchy-Riemann equations, Math. Sb. 99 (1976), 58-83.

16. W. Rudin, Function theory in the unit ball of $\mathbf{C}^{n}$, Springer-Verlag, New York, 1980.

17. M.-C. Shaw, Hölder and $L^{p}$ estimates for $\bar{\partial}_{b}$ on weakly pseudo-convex boundaries in $\mathbf{C}^{2}$, Math. Ann. 279 (1988), 635-652.

18. H. Skoda, Valeurs au bord pour les solutions de l'opérateur $d^{\prime \prime}$, et charactérisation des zéros des fonctions de la classe de Nevanlinna, Bull. Soc. Math. France 104 (1976), 225-299.

19. E. M. Stein, Singular integrals and estimates for the Cauchy-Riemann equations, Bull. Amer. Math. Soc. 75 (1973), 440-445.

20. __ Boundary behavior of holomorphic functions of several complex variables, Princeton Univ. Press, Princeton, N. J., 1972.

21. W. Stoll, Die beiden Hauptsätze der Wertverteilungstheorie bei Funktionen mehrerer komplexer Veränderlichen. I, Acta Math. 90 (1953), 1-115.

Department of Mathematics, University of Notre Dame, Notre Dame, Indiana 46556 